AperTO - Archivio Istituzionale Open Access dell'Università di Torino

\title{
Altered resting state attentional networks in diabetic neuropathic pain
}

\section{This is the author's manuscript}

Original Citation:

Availability:

This version is available http://hdl.handle.net/2318/76719

since

Published version:

DOI:10.1136/jnnp.2009.188631

Terms of use:

Open Access

Anyone can freely access the full text of works made available as "Open Access". Works made available under a Creative Commons license can be used according to the terms and conditions of said license. Use of all other works requires consent of the right holder (author or publisher) if not exempted from copyright protection by the applicable law. 


\section{(38) \\ UNIVERSITÀ DEGLI STUDI DI TORINO}

This is an author version of the contribution published on:

J Neurol Neurosurg Psychiatry (2010), 81(7):806-11

doi: 10.1136/jnnp.2009.188631

The definitive version is available at:

http://jnnp.bmj.com/content/early/2009/12/01/jnnp.2009.188631 


\title{
Altered resting state attentional networks in diabetic neuropathic pain
}

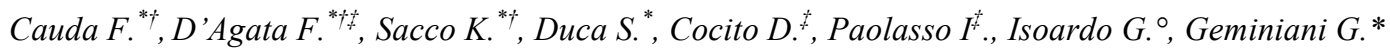 \\ ${ }^{*}$ CCS fMRI, Koelliker Hospital, Turin, Italy \\ ${ }^{*}$ Department of Psychology, University of Turin, Turin, Italy \\ * Department of Neuroscience, AOU San Giovanni Battista, Turin, Italy \\ Neurophysiology unit, Department of Neurosurgery, Azienda Sanitaria CTO, Turin, Italy

\section{Corresponding author} \\ Dr. Cauda Franco, Dipartimento di Psicologia, Via Po 14, 10123 Turin, Italy \\ (franco.cauda@unito.it) \\ The authors declare no conflict of interest.
}

\section{Running title}

Altered attentional networks in Neuropathic Pain

\section{Word and character counts}

Abstract: 188 words. Paper: 3400 words, figures 3 (color 3).

\author{
Abbreviations: \\ DAN, Dorsal Attentional Network; DMN, Default Mode Network; fMRI, functional Magnetic \\ Resonance Imaging; NP, neuropathic pain; rsFC Resting state functional connectivity; VAN, \\ Ventral Attentional Network;
}

\section{Keywords}

Neuropathic pain, resting state, functional connectivity, attentional networks, functional magnetic resonance imaging (fMRI)

\begin{abstract}
Chronic pain can be considered as a highly salient stimulus that continuously taxes the attentional and salience processing networks, thus interfering with cognitive abilities and, more specifically, consuming attentional resources. The aim of the paper was to explore whether and how diabetic neuropathic pain (NP) affects attentional networks. We sought to achieve this by investigating resting state functional connectivity (rsFC) in diabetic NP patients and comparing it with that of matched healthy controls. NP patients showed a widespread reduction of connectivity in both the dorsal and ventral attentional networks, as well as in the dorsal anterior cingulated cortex (ACC), typically implicated in salience processing. We also found a generalized reduction of the length of functional connections in the NP group: in all the examined networks, the Euclidian distance between connected voxels was significantly shorter in patients vs. controls. In sum, our work showed that in diabetic NP pain a parieto-fronto-cingulate network controlling attention to external stimuli is impaired. In line with previous studies, we conclude that chronic pain can disrupt the synchrony of a common pool of brain areas, involved in self monitoring, pain processing and salience detection.
\end{abstract}




\section{INTRODUCTION}

The nervous system is continuously bombarded by internal and extrapersonal stimuli. The priority is to identify the most relevant among these myriad of inputs. This capacity requires a system that can integrate highly processed sensory data with visceral, autonomic, and hedonic markers [1]. Pain is inherently salient, and it demands attention and cognitive resources, as it has to be evaluated in intensity and in other qualities. Thus, attention to a salient input such as pain can be viewed as the focusing of resources on a specific stimulus. While a growing number of studies have examined the relationships between attention and pain, and their neural correlates, few studies have addressed the issue of how pain engages and modifies attention. Indeed, the majority of studies to date have focused on the effects of attention on pain, while the effects of pain on attention are practically unexplored.

Lesion and neuroimaging studies employing non-painful stimuli have related attentional control to a network composed of parietal, frontal, and cingulate cortical regions [2]. Some studies have found similar patterns of cortical activation for both voluntary and involuntary attentional control mechanisms [3]. Similarly to other attentional tasks, pain engages a common group of brain regions that includes the posterior parietal, prefrontal, and anterior cingulate cortices [4]. Sharing common cognitive resources and neural correlates, pain and attention systems are likely to interact. Indeed, it has been demonstrated that experimental pain can decrease cognitive performance and task-related activity $[5,6]$. However, the interaction between pain and other functional networks has only recently started to be considered $[7,8]$.

We believe a promising perspective would be to consider the mechanisms underlying pain perception as a complex phenomenon that engages multiple cerebral areas acting together as a functional unit. Although not fully understood, such cognitive mechanisms have been shown to be impaired in patients suffering from chronic pain $[6,9,10]$. Thus, patients with chronic neuropathic pain may represent a suitable population among which to investigate the effects of pain on cognitive functions and, more specifically, to shed light on how brain networks involved in attention systems can be modified by pain.

Resting state functional connectivity (rsFC) is a network analysis approach, based on temporal correlations between the activity in a seed area and the rest of the brain. It considers a set of brain regions, assuming that areas working in unison show a high correlation between respective time courses. It has been proposed, from this viewpoint, that behavioral correlates might be represented better in patterns of connectivity than in regional activity. rsFC can reveal the underlying structure of biologically plausible and reproducible functional networks in several domains [11, 12]. rsFC is increasingly being used to study system organization in development $[13,14]$ and pathologies $[15$, 16]. Other studies have already demonstrated that brain rsFC is disrupted across different neuropsychiatric conditions [17], suggesting that the study of brain rsFC might be useful to understand disease states as well as potentially provide diagnostic information. Hence, rsFC MRI is well-suited to study the potential changes of brain system organization in patients with chronic pain. Interconnections between pain and attention-related areas have been shown, based on their common temporal activity patterns during rest: Ohara and colleagues [18] demonstrated that cortical areas with pain-related activity are functionally connected during attention to a painful stimulus. However, only a couple of studies [7, 19] have focused on how chronic pain can affect rsFC, demonstrating that long-term pain can disrupt the Default Mode Network (DMN) [20] rsFC, i.e. the functional interconnections among brain regions characterized by high baseline activity that decreases during attention-demanding cognitive tasks. Furthermore, Seminowicz and colleagues [8] demonstrated that experimental pain can alter the functional connectivity evoked by an attentional task. Overall, these studies show that pain can alter the functional connectivity of networks involved in pain and homeostatic processing and thus they suggest that chronically taxed attentional networks can suffer and become impaired after years of continuous chronic pain. In the present study, we intend to investigate in more detail how and which attention systems can be affected by chronic pain, using the functional connectivity method. 
Fox and colleagues [21] demonstrated a bilateral dorsal attention system involved in top-down orienting of attention and a right-lateralized ventral attention system involved in reorienting attention in response to salient sensory stimuli. These two networks had previously been visualized in task-induced activation studies (for a review see [22]). The integrity of both Dorsal Attentional Network (DAN) and Ventral Attentional Network (VAN) rsFC has been explored in patients with spatial neglect, in whom a common disruption of specific areas of DAN and VAN was found.[23] We thus considered these two attention systems and their possible modifications following chronic pain.

\section{METHODS}

For a more detailed description see the supplementary online materials.

\section{Patients}

Eight right-handed patients suffering from peripheral Neuropathic Pain (NP) (four women and four men suffering from diabetic pain; age range $=51-78$, mean age $=61$ years) were enrolled from our multidisciplinary pain unit (Table S1). This group of patients was also the subject of a previous study [19 ]. All patients underwent a complete neurological and psychological examination as well as standard MR brain scanning by an experienced Neuroradiologist (SD) to exclude structural/white-matter abnormalities on anatomical MR-images. Patients suffering from significant central nervous disease and psychiatric disorders were excluded. All patients were assessed using standardized pain scales (visual analog scale - VAS, numerical rating scale - NRS, McGill Pain Questionnaire - MPQ, Italian version). The spontaneous component of their pain syndrome was evaluated on the MPQ checklist. VAS readings were obtained from their clinical records and both the day before and on the day of the study. In all cases pain was restricted to the bilateral lower limbs. Duration of pain was $>2$ years in all cases. Patients were washed out of their medications at least one month before imaging (opioids or cannabinoids were never administered). Maximum care was taken to avoid situations that could actually trigger evoked pain during the imaging sessions.

Eight age- and gender-matched right-handed healthy volunteers (four women and four men; age range $=47-79$, mean age $=59$ years) acted as controls. None suffered from any neurological or psychiatric disorders, including chronic pain of any kind, nor had a history of drug or alcohol abuse. None were on medications known to alter brain activity.

All subjects gave their informed written consent, in line with the Declaration of Helsinki, and the study was approved by the local ethics committee.

\section{Procedures}

All subjects were instructed simply to keep their eyes closed, to think of nothing in particular, and not to fall asleep. After the scanning session, participants were asked if they had fallen asleep during the scan; those who provided a positive or dubious answer were excluded from the study.

Data acquisition was performed on a 1.5 Tesla INTERA ${ }^{\mathrm{TM}}$ scanner (Philips Medical Systems) with a SENSE high-field, high-resolution (MRIDC) head coil optimized for functional imaging. Resting state functional T2*-weighted images were acquired using echoplanar (EPI) sequences; in the same session, a set of three-dimensional high-resolution $\mathrm{T}_{1}$-weighted structural images was acquired for each participant (see supplementary material for sequences' details). Blood oxygen level dependent (BOLD) imaging data were analyzed using the Brain Voyager QX software (Brain Innovation, Maastricht, Holland). In order to reduce artifacts functional images were pre-processed as follows: (1) Slice scan time correction; (2) 3D motion correction; (3) Spatial smoothing (Gaussian kernel of $8 \mathrm{~mm}$ FWHM); (4) Temporal filters (linear trend removals) were used to reduce cardiac as well as respiratory noise, and a band pass filter of $0.01-0.08 \mathrm{~Hz}$ was used as several previous studies [24, 25] have found the frequency range $[0.01-0.08 \mathrm{~Hz}]$ to have the greatest power in revealing the underlying connectivity $[14,24]$. After pre-processing, each subject's slice-based functional scan 
was co-registered with their 3D high-resolution structural scan and transformed into Talairach space. Functional connectivity was measured using a method similar to [23] as described below:

ROIs for functional connectivity analyses of dorsal attention network (DAN) and ventral attention network (VAN) were determined from a meta analysis of four previously published event-related fMRI studies of adults performing an attentional paradigm as previously suggested in the paper by He et al. [23]. The studies used for this meta-analysis were: Astafiev et al. and Corbetta et al. [26, 27]. We defined eight ROIs $\left(512 \mathrm{~mm}^{3}\right.$ each) representing the DAN and five regions $\left(512 \mathrm{~mm}^{3}\right.$ each) representing the VAN. To further investigate the attentional system we added a couple of supplemental ROIs $\left(216 \mathrm{~mm}^{3}\right.$ each) in the bilateral dorsal cingulated (dACC) cortex, selected according to Margulies et al. [28]. All selected ROIs are listed in Table S2.

The first step in all rsFC analyses was to extract BOLD time courses from each ROI by averaging over voxels within each region. To compute rsFC maps corresponding to a selected seed ROI, the regional time course was correlated against all other voxels within the brain. The maps, representing the connectivity of a network (DAN, VAN) or dACC, were computed by conjunction analysis of the selected ROIs.

The individual participant multiple regression analysis (carried out using the general linear model (GLM)) resulted in a t-based map corrected for multiple comparisons with the false discovery rate (FDR $\mathrm{q}<0.05$, Cluster threshold $\mathrm{K}>5$ voxels in the native functional resolution). Several nuisance covariates were included in our analyses to control for the effects of physiological processes and motion. Specifically, we included nine additional covariates that modeled nuisance signals from WM, cerebrospinal fluid (CSF) and global signal (GS), as well as six motion parameters.

Random effect group-level analyses, controlling for age and gender effects, were conducted using the GLM and the ANCOVA analysis tool implemented in BrainVoyager QX. Corrections for multiple comparisons were carried out for each of the two networks and for the dACC using the FDR ( $q<0.05$, cluster threshold $>5$ voxels in the native resolution). To test for significance of grouprelated differences, direct voxelwise group comparisons were performed using group-level contrasts. These contrasts produced FDR thresholded maps $(\mathrm{q}<0.05$, Cluster threshold $\mathrm{K}>5$ voxels in the native resolution) of those voxels that showed significant differences between the two groups. The resulting maps were then projected on a flattened representation of the brain using the BrainVoyager QX surface tool. Probabilistic maps were computed to evaluate the spatial consistency of functional connectivity patterns across subjects.

For each network (DAN or VAN), plus the dACC, and for each subject, we calculated the Euclidean distance between the center of gravity of all centers of the respective ROIs and every other voxel that reached significance in the thresholded Z-score map of positive rsFC (cluster significance: $\mathrm{p}<0.05$, minimum cluster size $\mathrm{k}>5$ contiguous voxels). We examined group differences by performing a parametric analysis (t-tests for independent samples, patients vs. controls) for the total number of active voxels within $120 \mathrm{~mm}$.

To test our results and demonstrate that our findings were not linked to some sort of overall effect in all the voxels of the patient group data, we calculated the rsFC of two more networks and compared the patient and control groups (see supplementary online materials and results).

\section{RESULTS}

\section{Probabilistic maps}

The spatial probability maps of the three rsFC networks showed good spatial reproducibility of individual subjects' results (see Fig S5 and Table S3, S4, S5) and very similar spatial distribution as previous rsFC studies of the same networks.[21,28]

\section{Functional connectivity in DAN and VAN}

The rsFC comparison of the two groups showed reduced connectivity in the DAN as well as in the VAN for pain subjects. Voxelwise group comparisons revealed differences in both networks (see 
Fig 1, S8, S9 and Table S9, S10); the cumulative voxel count (see Fig S7) confirmed the prevalence of the rsFC mean number of voxels in the DAN and in the VAN for healthy subjects.

The dACC connectivity maps (see Fig 1, S10 and Table S11) replicated the evidence of reduced connectivity seen in the DAN and VAN; as expected, the cumulative voxel count (see Fig S7) confirmed the results of the voxelwise analysis.

\section{Overlapping between maps}

To identify the degree of overlapping between all three rsFC subtraction maps we performed an intersection analysis of the maps displayed in Fig 1, S8, S9, S10 and summarized in Table S9, S10, S11. This yielded the areas of overlapping of the subtraction maps for two networks (DAN and VAN): in the bilateral Middle Temporal Gyrus, bilateral Superior Temporal Gyrus, bilateral Cingulate Gyrus (dACC), bilateral Middle Frontal Gyrus (Supplementary Motor Area and Frontopolar Area) and right Cuneus, and for three networks (DAN, VAN and additionally dACC): in the bilateral Superior Temporal Gyrus, left Cingulate Gyrus (dACC) and right Cuneus (see Fig 2, S11, S12 and Table S7, S8).

Insert Fig 1 and Fig 2 about here

To identify nodes that were common to both networks (DAN and VAN) and dACC we performed a conjunction analysis between normal rsFC for all healthy subjects. Potential hub areas were found in the bilateral Cingulate Gyrus, right Precentral Gyrus, right Supramarginal Gyrus, left Middle Frontal Gyrus (see Fig S6 and Table S8).

\section{Voxel-distance differences}

A significant decrease in the numbers of connected voxels was found in the patient group compared to the control group (see Fig 3).

\section{Insert Fig 3}

\section{DISCUSSION}

The present fMRI data suggest that in diabetic neuropathic subjects there is a reduction in attentional network rsFC. The networks investigated in our study are superimposable on those described in previous papers [22]. We found limited areas of overlapping between the networks being studied as described in a previous study by Fox and colleagues [21]. The comparison of the DAN and VAN, respectively, in patients and healthy subjects, showed a widespread reduction in rsFC in both the attentional networks. These results seem to indicate a disruption of the attentional and salience processing networks [21].

The paper by Seminowicz et al. [8] indicates that pain can modulate a network involved in focused attention, suggesting a mechanism for the interference of pain on cognitive ability by the consumption of attentional resources. This interpretation is consistent with our data: chronic pain is a highly salient stimulus, continuously taxing the attentional and salience processing networks and which could modify the activity of those networks. This idea is supported by the findings of Baliki et al. [7]: they demonstrated that chronic pain has a widespread impact on overall brain function, 
suggesting that the disruptions of the DMN [29] which they reported may underlie the cognitive and behavioral impairments accompanying chronic pain $[6,10]$. These findings were confirmed by our recent study [19 ] in which we observed reduced DMN connectivity in the bilateral primary sensorimotor areas and cingulated cortex, as well as left temporal and occipital cortices during resting states.

Our study revealed a reduction or disruption of some resting state functional networks in the pathological population we examined. Of note, similar alterations of resting state network dynamics have been demonstrated in a number of neuropsychiatric disorders, including, Alzheimer's disease, epilepsy, depression, schizophrenia, clinical conditions characterized by impaired attention, such as attention deficit hyperactivity disorder and neglect. Although rsFC has been widely used, to date only one study [23] has explored how a neuropsychological syndrome specifically involving attention (spatial neglect) is related to changes in attentional networks. This study, using a methodology similar to ours, found an acute disruption and disrupted connectivity in specific pathways in the dorsal and ventral networks that strongly correlated with impaired attentional processing across subjects.

To further investigate the attentional networks we added two additional ROIs in the anterior Cingulate Cortex. In their study Marguiles [28] and colleagues placed a series of small ROIs in the anterior Cingulated Cortex and found a location positively correlated with regions typically implicated in attentional processes (e.g., dorsolateral and posterior inferior prefrontal cortices). This region has also been observed to be implicated in "salience processing" including the emotional aspects of pain.[1] The dACC plays a role in the response to varied forms of salience, including the emotional dimensions of pain [32] and empathy for pain [33]. The comparison between patients and healthy subjects led to similar results for all the networks explored (DAN, VAN), and also to the observation of a significant superposition of areas with reduced rsFC. Several of those areas were also found to have reduced $\mathrm{rsFC}$ in a previous study exploring modifications in resting state functional connectivity subsequent to pain [7]. The fact that all the networks examined in this work revealed a pattern of reduced connectivity and that this pattern is partially superimposable on the findings of previous studies on DMN rsFC [29] suggests that chronic pain could disrupt the synchrony of a common pool of brain areas involved in self monitoring, pain processing and salience detection that can be explored using several different functional connectivity techniques. Chronic pain-induced cortical/subcortical reorganization has been documented in animal models [30] as well as in humans [31]. More specifically, the reduced connectivity in the prefrontal cortex observed in our study is consistent with recent findings. Metz et al. [34] found that in rodents neuropathic pain leads to a rearrangement of the prefrontal cortex; Ploner et al. demonstrated that pain, in healthy controls, has the unique capability of disrupting the resting electroencephalogram (EEG) rhythm on a large-scale spatial dimension (i.e. both alpha on the occipital areas and murhythm on the sensorymotor areas [35]).

The analysis of differences in voxel distances indicates a generalized reduction of connected voxels in the patient group in all the networks and in the AACC. That reduction is in line with several other results showing that a number of pathologies have a widespread impact on the brain network structure and efficiency (for a review see Guye et al.[36]).

Our findings support a crucial role of the dorsal ACC in attentional network modulation. Fox et al. [21] suggested that the prefrontal cortex (middle and inferior frontal gyrus) may be a locus of functional interaction between the ventral attention system and the dorsal attention system. Our analysis of overlapping areas suggests that dorsal ACC may play a pivotal role in such an interaction. Gitelman [3] and Mesulam [37] hypothesized that spatial attention to external stimuli is supported by a large-scale network connecting not only the fronto-parietal cortices, but also the cingulated cortex; such a network may support mental representation of motivational salience of external stimuli. Chronic pain could modulate the reduced activation of the parieto-fronto-cingulate network through the anterior cingulated cortex. This hypothesis seems to be supported by the 
extensively reduced connectivity of the dorsal ACC (see fig 1 and 2) and by the reduction in the number of significantly positively correlated voxels at long distances ( $>60 \mathrm{~mm}$ ) (see figure S7).

Several studies have shown that the ACC is a crucial area involved in the attentional modulation of pain, Bantick et al. [38] found that subjects performing an attentional task (Stroop test) during painful stimulation showed reduced pain perception which was related to activation in areas of the "pain matrix", including the dorsal ACC. On the other hand, the crucial role of the ACC in affective modulation of pain is supported by several studies; a meta analysis by Peyron et al. [32] showed the ACC to be involved in both affective and attentional modulation of pain perception and not in modulation of simple perceptive aspects of pain (such as intensity and localization of pain). Experimental studies on the modulation of pain by hypnotic suggestion found the ACC to be the locus of interaction between sensory and affective modulation of pain.[39]

One could hypothesize about different mechanisms to explain the reduced connectivity in chronic pain (overcompensation of chronic excitation, toxic/degenerative processes, cognitive plasticity related to learning processes), but our study did not aim to investigate this point.

\section{Limitations of the study and future research}

This study enrolled only a limited number of patients, belonging to a specific neuropathic condition (diabetic patients). Further research should explore the impact of chronic pain on DMN/ attentional systems in other clinical populations: possible interactions with etiology, concomitant symptoms, staging and grading of pain perception should be examined.

\section{ACKNOWLEDGEMENTS}

We wish to thank all the subjects who participated in this study. This research was supported by the Italian Ministry of Education, University and Research (MIUR), PRIN project 2008, prot. 2007P2CWAZ.

We declare no competing interests.

The Corresponding Author has the right to grant on behalf of all authors and does grant on behalf of all authors, an exclusive licence on a worldwide basis to the BMJ Publishing Group Ltd and its Licensees to permit this article (if accepted) to be published in Journal of Neurology, Neurosurgery $\&$ Psychiatry and any other BMJPGL products to exploit all subsidiary rights. 


\section{REFERENCES}

1. Damasio AR. How the brain creates the mind. Sci Am. 1999;281(6):112-7.

2. Corbetta M, Miezin FM, Dobmeyer S, et al. Selective and divided attention during visual discriminations of shape, color, and speed: functional anatomy by positron emission tomography. $\mathrm{J}$ Neurosci. 1991;11(8):2383-402.

3. Gitelman DR, Nobre AC, Parrish TB, et al. A large-scale distributed network for covert spatial attention: further anatomical delineation based on stringent behavioural and cognitive controls. Brain. 1999; 122:1093-106.

4. Naghavi HR, Nyberg L. Common fronto-parietal activity in attention, memory, and consciousness: shared demands on integration? Conscious Cogn. 2005;14(2):390-425.

5. Houlihan ME, McGrath PJ, Connolly JF, et al. Assessing the effect of pain on demands for attentional resources using ERPs. Int J Psychophysiol. 2004;51(2):181-7.

6. Lorenz J, Beck H, Bromm B. Cognitive performance, mood and experimental pain before and during morphine-induced analgesia in patients with chronic non-malignant pain. Pain. 1997;73(3):369-75.

7. Baliki MN, Geha PY, Apkarian AV, et al. Beyond feeling: chronic pain hurts the brain, disrupting the default-mode network dynamics. J Neurosci. 2008;28(6):1398-403.

8. Seminowicz DA, Davis KD. Pain enhances functional connectivity of a brain network evoked by performance of a cognitive task. J Neurophysiol. 2007;97(5):3651-9.

9. Dick B, Eccleston C, Crombez G. Attentional functioning in fibromyalgia, rheumatoid arthritis, and musculoskeletal pain patients. Arthritis Rheum. 2002;47(6):639-44.

10. Veldhuijzen DS, Kenemans JL, van Wijck AJ, et al. Processing capacity in chronic pain patients: a visual event-related potentials study. Pain. 2006;121(1-2):60-8.

11. Dosenbach NU, Fair DA, Miezin FM, et al. Distinct brain networks for adaptive and stable task control in humans. Proc Natl Acad Sci U S A. 2007;104(26):11073-8.

12. Fox MD, Raichle ME. Spontaneous fluctuations in brain activity observed with functional magnetic resonance imaging. Nat Rev Neurosci. 2007;8(9):700-11.

13. Fair DA, Cohen AL, Dosenbach NU, et al. The maturing architecture of the brain's default network. Proc Natl Acad Sci U S A. 2008;105(10):4028-32.

14. Fransson P. Spontaneous low-frequency BOLD signal fluctuations: an fMRI investigation of the resting-state default mode of brain function hypothesis. Hum Brain Mapp. 2005;26(1):15-29.

15. Castellanos FX, Margulies DS, Kelly C, et al. Cingulate-precuneus interactions: a new locus of dysfunction in adult attention-deficit/hyperactivity disorder. Biol Psychiatry. 2008;63(3):332-7.

16. Greicius MD, Flores $\mathrm{BH}$, Menon V, et al. Resting-state functional connectivity in major depression: abnormally increased contributions from subgenual cingulate cortex and thalamus. Biol Psychiatry. 2007;62(5):429-37.

17. Greicius M. Resting-state functional connectivity in neuropsychiatric disorders. Curr Opin Neurol. 2008;21(4):424-30.

18. Ohara S, Crone NE, Weiss N, et al. Analysis of synchrony demonstrates 'pain networks' defined by rapidly switching, task-specific, functional connectivity between pain-related cortical structures. Pain. 2006;123(3):244-53.

19. Cauda F, Sacco K, Duca S, et al. Altered resting state in diabetic neuropathic pain. PLoS ONE. 2009;4(2):e4542.

20. Raichle ME, Snyder AZ. A default mode of brain function: a brief history of an evolving idea. Neuroimage. 2007;37(4):1083-90; discussion 97-9.

21. Fox MD, Corbetta M, Snyder AZ, et al. Spontaneous neuronal activity distinguishes human dorsal and ventral attention systems. Proc Natl Acad Sci U S A. 2006;103(26):10046-51.

22. Corbetta M, Shulman GL. Control of goal-directed and stimulus-driven attention in the brain. Nat Rev Neurosci. 2002;3(3):201-15. 
23. He BJ, Snyder AZ, Vincent JL, et al. Breakdown of functional connectivity in frontoparietal networks underlies behavioral deficits in spatial neglect. Neuron. 2007;53(6):905-18.

24. Biswal B, Yetkin FZ, Haughton VM, et al. Functional connectivity in the motor cortex of resting human brain using echo-planar MRI. Magn Reson Med. 1995;34(4):537-41.

25. Greicius MD, Krasnow B, Reiss AL, et al. Functional connectivity in the resting brain: a network analysis of the default mode hypothesis. Proc Natl Acad Sci U S A. 2003;100(1):253-8.

26. Astafiev SV, Stanley CM, Shulman GL, et al. Extrastriate body area in human occipital cortex responds to the performance of motor actions. Nat Neurosci. 2004;7(5):542-8.

27. Corbetta M, Kincade JM, Ollinger JM, et al. Voluntary orienting is dissociated from target detection in human posterior parietal cortex. Nat Neurosci. 2000;3(3):292-7.

28. Margulies DS, Kelly AM, Uddin LQ, et al. Mapping the functional connectivity of anterior cingulate cortex. Neuroimage. 2007;37(2):579-88.

29. Raichle ME, MacLeod AM, Snyder AZ, et al. A default mode of brain function. Proc Natl Acad Sci U S A. 2001;98(2):676-82.

30. Julius D, Basbaum AI. Molecular mechanisms of nociception. Nature. 2001;413(6852):203-

10.

31. Sorensen L, Siddall PJ, Trenell MI, et al. Differences in metabolites in pain-processing brain regions in patients with diabetes and painful neuropathy. Diabetes Care. 2008;31(5):980-1.

32. Peyron R, Laurent B, Garcia-Larrea L. Functional imaging of brain responses to pain. A review and meta-analysis (2000). Neurophysiol Clin. 2000;30(5):263-88.

33. Singer T, Seymour B, O'Doherty J, et al. Empathy for pain involves the affective but not sensory components of pain. Science. 2004;303(5661):1157-62.

34. Metz AE, Yau HJ, Centeno MV, et al. Morphological and functional reorganization of rat medial prefrontal cortex in neuropathic pain. Proc Natl Acad Sci U S A. 2009;106(7):2423-8.

35. Ploner M, Gross J, Timmermann L, et al. Pain suppresses spontaneous brain rhythms. Cereb Cortex. 2006;16(4):537-40.

36. Guye M, Bartolomei F, Ranjeva JP. Imaging structural and functional connectivity: towards a unified definition of human brain organization? Curr Opin Neurol. 2008;21(4):393-403.

37. Mesulam MM. Spatial attention and neglect: parietal, frontal and cingulate contributions to the mental representation and attentional targeting of salient extrapersonal events. Philos Trans $\mathrm{R}$ Soc Lond B Biol Sci. 1999;354(1387):1325-46.

38. Bantick SJ, Wise RG, Ploghaus A, et al. Imaging how attention modulates pain in humans using functional MRI. Brain. 2002;125(Pt 2):310-9.

39. Rainville P, Duncan GH, Price DD, et al. Pain affect encoded in human anterior cingulate but not somatosensory cortex. Science. 1997;277(5328):968-71. 


\section{CAPTIONS}

\section{Figure 1 DAN, VAN and dACC rsFC group comparison}

Two sample t-test $\mathrm{q}<0.05$ FDR-corrected, minimum cluster dimension $\mathrm{K}>5$ voxels in the native resolution. Colors from red to yellow indicate increased connectivity. Colors from blue to green indicate reduced connectivity. All rsFC maps were projected on a flattened representation of the brain using BrainVoyager QX.

\section{Figure 2 Overlapping of DAN, VAN and dACC rsFC group comparison}

Green, red and blue indicate, respectively, reduced connectivity in the DAN, VAN and dACC. Areas of overlapping are highlighted. Two sample t-test $\mathrm{q}<0.05$ FDR-corrected, minimum cluster dimension $\mathrm{K}>5$ voxels in the native resolution. All rsFC maps were projected on a flattened representation of the brain using BrainVoyager QX.

\section{Figure 3 Cumulative voxel count of DAN, VAN and dACC}

Cumulative voxel count of global significantly positively correlated voxels for DAN, VAN and dACC (from left to right); red bars for patients, blue for controls, asterisks show significant differences ( $\mathrm{t}$-tests for independent samples, patients vs. controls $\mathrm{p}<0.05$ ). 